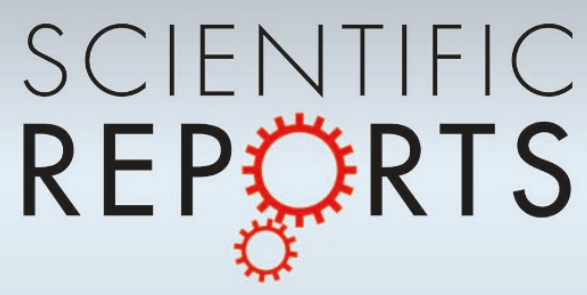

OPEN

SUBJECT AREAS:

NANOMETROLOGY

IMAGING TECHNIQUES

NANOPARTICLES

CHARACTERIZATION AND

ANALYTICAL

TECHNIQUES

Received

18 March 2013

Accepted

26 June 2013

Published

19 July 2013

Correspondence and requests for materials should be addressed to Y.C.S. (ycsasaki@k.utokyo.ac.jp)

\section{Tracking 3D Picometer-Scale Motions of Single Nanoparticles with High-Energy Electron Probes}

\author{
Naoki Ogawa ${ }^{1,2}$, Kentaro Hoshisashi ${ }^{1,3}$, Hiroshi Sekiguchi ${ }^{1,4}$, Kouhei Ichiyanagi i, ${ }^{1,3}$, Yufuku Matsushita ${ }^{3}$, \\ Yasuhisa Hirohata ${ }^{1,2}$, Seiichi Suzuki ${ }^{5}$, Akira Ishikawa ${ }^{1,2} \&$ Yuji C. Sasaki ${ }^{1,3,4}$
}

'JST/CREST SASAKI-team, Japan Science and Technology Agency, The University of Tokyo, 5-1-5 Kashiwanoha, Kashiwa City, Chiba, JAPAN, ${ }^{2}$ Department of Physics, College of Humanities and Sciences, Nihon University, 3-25-40 Sakurajosui, Setagaya-Ku, Tokyo, JAPAN, ${ }^{3}$ Graduate School of Frontier Sciences, The University of Tokyo, 5-1-5 Kashiwanoha, Kashiwa City, Chiba, JAPAN, ${ }^{4}$ Research \& Utilization Div., Japan Synchrotron Radiation Research Institute, SPring-8 1-1-1 Kouto, Sayo-cho, Sayo-gun, Hyogo, JAPAN, ${ }^{5}$ TSL Solutions K. K., Sagamihara, Kanagawa, JAPAN.

We observed the high-speed anisotropic motion of an individual gold nanoparticle in 3D at the picometer scale using a high-energy electron probe. Diffracted electron tracking (DET) using the electron back-scattered diffraction (EBSD) patterns of labeled nanoparticles under wet-SEM allowed us to super-accurately measure the time-resolved 3D motion of individual nanoparticles in aqueous conditions. The highly precise DET data corresponded to the 3D anisotropic log-normal Gaussian distributions over time at the millisecond scale.

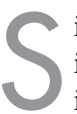

ingle-molecule observation techniques using visible-wavelength fluorescent probes are valuable for answering many basic and important questions in biology and biophysics because they provide the positional information of single molecules with accuracy far beyond the half-wavelength optical diffraction limit. They can provide the center positional information of labeled molecules with an accuracy of approximately $\lambda /$ $100^{1,2}$. Using similar tracking techniques with $\mathrm{X}$-ray $(\lambda \approx 0.1 \mathrm{~nm})$ probes, we have previously observed the picometer-scale $(\lambda / 100)$ Brownian motions of individual protein molecules linked to gold nanocrystals ${ }^{3}$. This system for single-molecule observation is called diffracted X-ray tracking (DXT) ${ }^{4}$. In this study, instead of using $\mathrm{X}$-rays, we used electron probes to improve this technology to develop a laboratory-scale single-molecule detection system.

Wet scanning electron microscopy (wet SEM) has recently been used to probe the cytoplasm of whole cells using a thin membranous partition that protects the sample from high-vacuum conditions. This method allows images of living cells to be captured with little loss of resolution compared to standard SEM. Wet SEM can also detect the internal motion of protein molecule domains in aqueous solutions on the sub-Angstrom scale at high rates of image acquisition ${ }^{5,6}$.

Because gold nanoparticles are highly electron dense, they are ideal for both transmission electron microscopy (TEM) and SEM. Colloidal gold has been recognized as an excellent marker for use in cell biology and materials science studies ${ }^{7}$. DXT allows direct picometer-scale observation of individual biomolecules in real time and in real space $^{8-10}$.

Figure 1(a) shows the arrangement of our new dynamic single-molecule detection system. Our system uses wet SEM and time-resolved electron backscatter diffraction (EBSD) from labeled gold nanoparticles. We refer to our new imaging technique as diffracted electron tracking (DET). A cross-sectional view of the wet cell used for DET is shown in Figure 1(b). We can measure the dynamics of both the top-layer of the adsorbed polymer reagent and the labeled gold nanoparticle. As a preliminary experiment, gold nanocrystals were directly adsorbed to the carbon surface for measurement by DET. As a result, we did not observe changes in the gold nanocrystal DET data over time. Rather, in this case we measured the movement of the coating polymer, which dictated the nanocrystal movement. Figure S1 shows an enlarged image of a sample cross-section. Because gold nanocrystals are slightly larger (diameter $=40 \mathrm{~nm}$ ), their movements do not represent individual single molecule movements, but rather, indicate the average movements of the nanoscale domains of the coating polymer. 


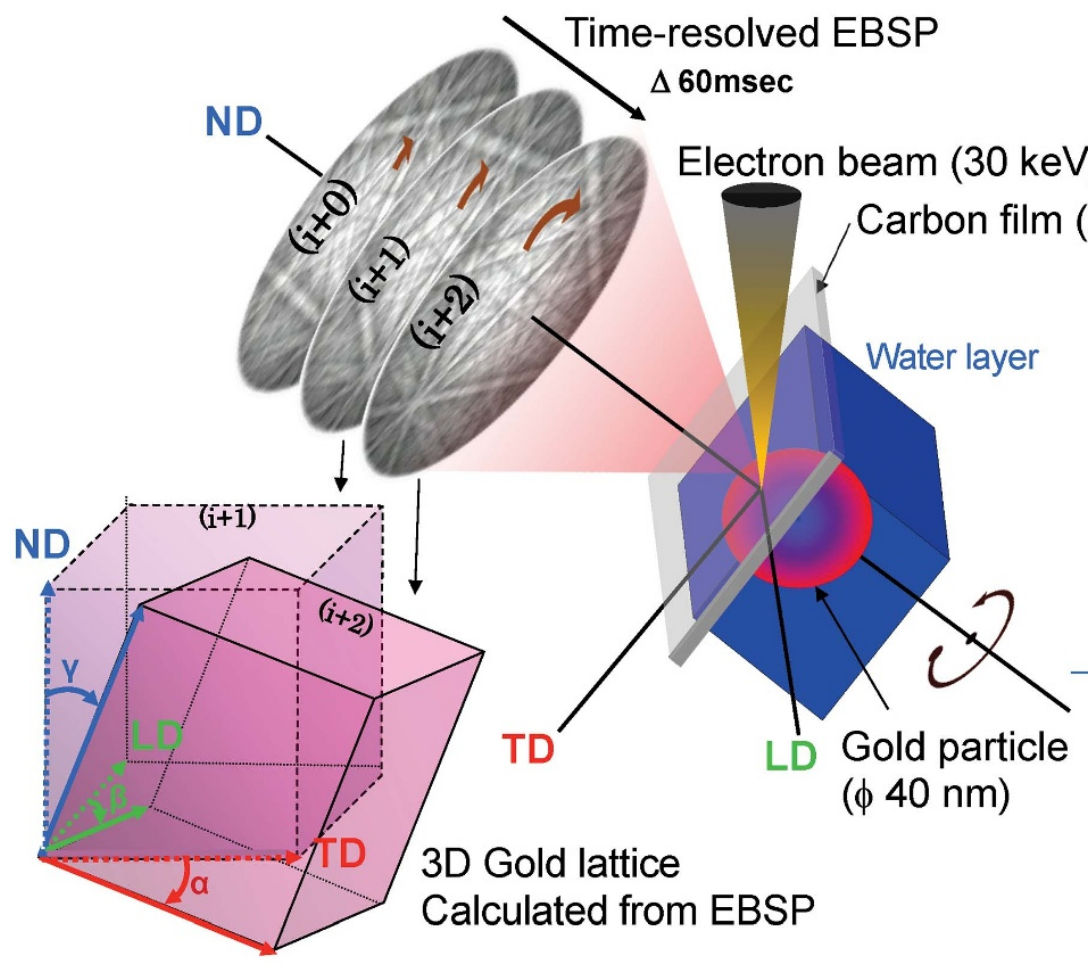

(a)
Electron probe $(30 \mathrm{KeV})$
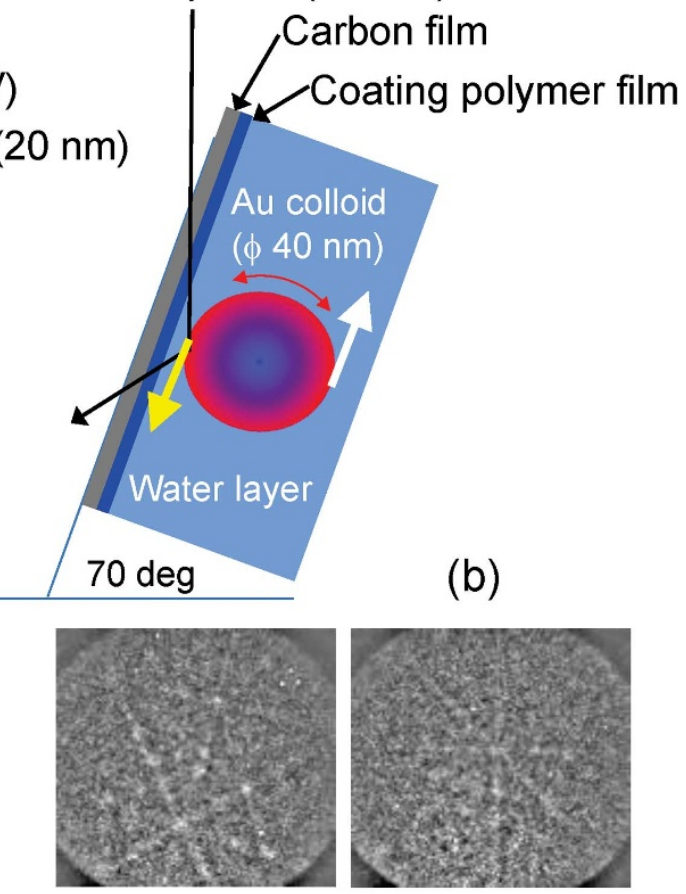

(c)

Figure $1 \mid$ (a) Conceptual diagram of Diffracted Electron Tracking (DET). This is the arrangement of our new dynamic single molecular detection system using the time-resolved EBSD pattern from the labeled gold nanoparticles on the coating polymer film with wet-SEM. DET can observe 3D dynamical motions of the labeled gold nanoparticles in the vacuum and aqueous solutions with the milli-second time scale. Longitudinal direction (LD), transversal direction (TD) and a normal direction (ND) are assigned to orientation representation in gold lattice from EBSD. (b) The cross-sectional view of the wet-cell for DET observations. In this case, we can observe the dynamical motions (each arrow) in coating polymer film (yellow) and labeled gold nanoparticle (white). Since the 3D dynamics of the labeled gold nanoparticle is measured from EBSD pattern from the labeled nanoparticle, we can extrapolate 3D movement of the top layer in the adsorbed polymer film. Here, the vacuum condition means that a water layer does not exist in a cell. (c) Typical EBSD pattern from gold nanoparticles (diameter size $=40 \mathrm{~nm}$ ) under the aqueous solution in the intergraded time of $60 \mathrm{~ms}$. Since the surface crystallinity of the gold nanoparticle is very good, the EBSD can detect gold nanoparticles in short integrated time from the aqueous solution. DET becomes a strong advantage over DXT when considering the size and convenience that comes with utilizing a marketed nanoparticles probe.

Because DXT uses X-rays, it requires the use of a perfect crystal. Therefore, for DXT, it is important to use gold nanocrystals instead of gold nanoparticles (colloidal gold). However, we can use commercially available gold nanoparticles for DET because the electron probe is sensitive to their surface structure. We confirmed from the electron diffraction data that the surface crystallinity of the gold nanoparticles was very good. As shown in Figure 1(c), EBSD patterns for the gold nanoparticles can be obtained with an integration time of only $60 \mathrm{~ms}$.

DET data collection is a simple process. First, we check the positions of the adsorbed gold nanoparticle using SEM, as shown Figure 2. Next, we collect time-resolved EBSD measurements. The direction of each EBSD measurement is determined in three dimensions for each time point. The orientation displacements at each time point can be obtained by comparing differences in the EBSD from the first crystallographic orientation pattern.

\section{Results}

The 3D (or 3-axis) motions of individual adsorbed gold nanoparticles within a vacuum are shown in Figure 3(a). The rotational motions around the $\beta$-axis were slightly smaller than the rotational motions around the $\alpha$ - and $\gamma$-axes. In the vacuum, the rotational motion velocities around the $\alpha$ - and $\gamma$-axes were approximately $0.05 \mathrm{rad} / \mathrm{s}$.

The 3D motion data for aqueous solutions are shown in Figure 3(b). The Brownian motion of the adsorbed gold nanoparticles in water was much more energetic than under vacuum. The rotational motions around each of the 3 axes were on the same order. In water, the rotational motion velocities around the $\alpha$ - and $\beta$-axes were approximately $0.55 \mathrm{rad} / \mathrm{s}$.

To understand the motion under vacuum and in solution, we analyzed and characterized the random displacements of individual single gold nanoparticles. The distribution of the displacement measurements observed using a $600 \mathrm{~ms}$ (ten 60 -ms frames) time interval is shown in Figure 4(a). As shown in Figure 4(b), the lognormal distributions can be characterized by the average $(\mu)$ and standard deviation $(\sigma)$ of the symmetrical normal Gaussian probability obtained when the displacements are plotted on logarithmic scales ${ }^{11}$. We confirmed that the log-normal distributions were similar at different time intervals (from 2 to 20 frames).

We determined the $\mu$ and $\sigma$ values from our distributions by fitting the statistical parameters with a Gaussian function as shown in Figure 4(b). The minimum value of $\mu$ under vacuum was assigned to the $\beta$-axis. This means that under vacuum conditions, the overall motions of the gold nanoparticles were rotational, thus defining the central axis as the LD axis (Fig. 1(a)).

The nanoparticle motions in aqueous solutions were 5-7 times larger than those in vacuum. We can hypothesize that this enhanced motion was due to collisions between the water molecules and the gold nanoparticles. The minimum value of $\mu$ under aqueous conditions was assigned to the $\gamma$-axis. This means that under aqueous conditions, the overall gold nanoparticle motions were rotational, thus defining the central axis as the ND axis (Fig. 1(a)). 


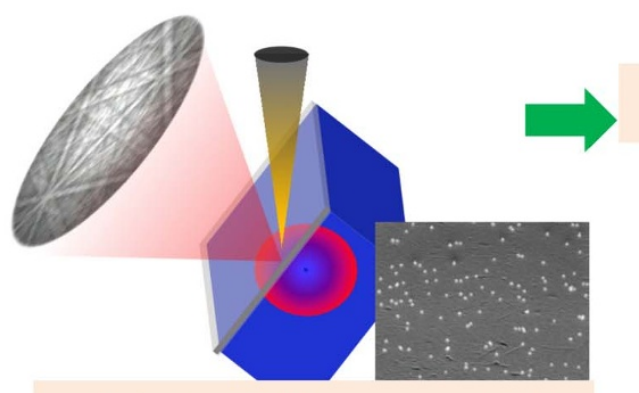

1.Select Nanocrystal

\section{Point time-resolved EBSD}
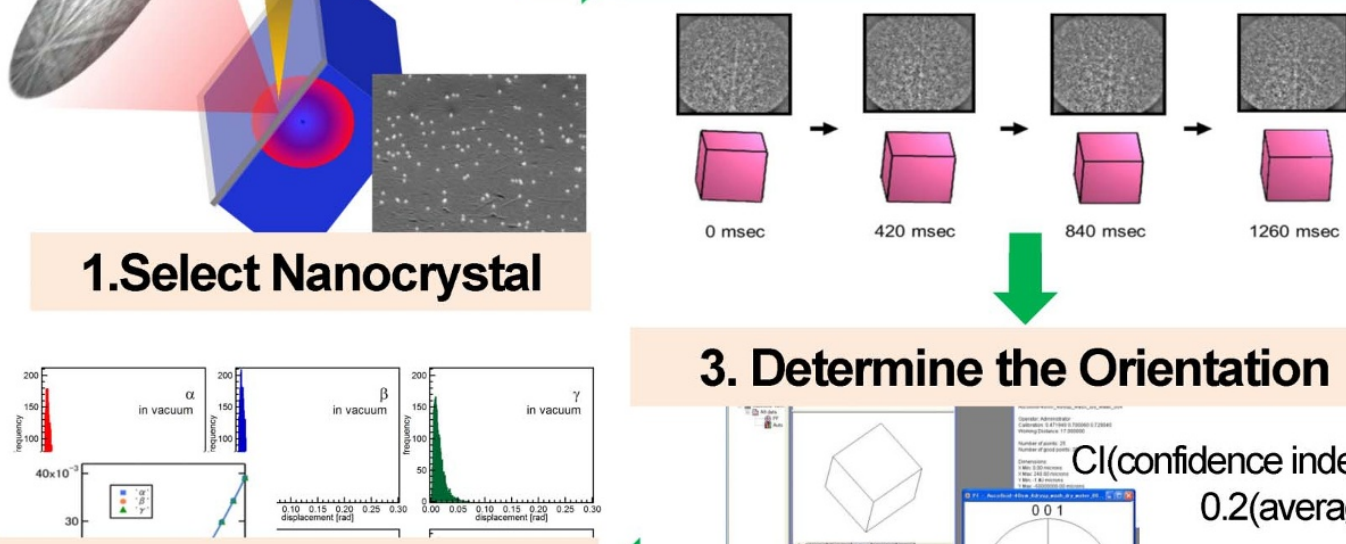

\section{Determine the Orientation}
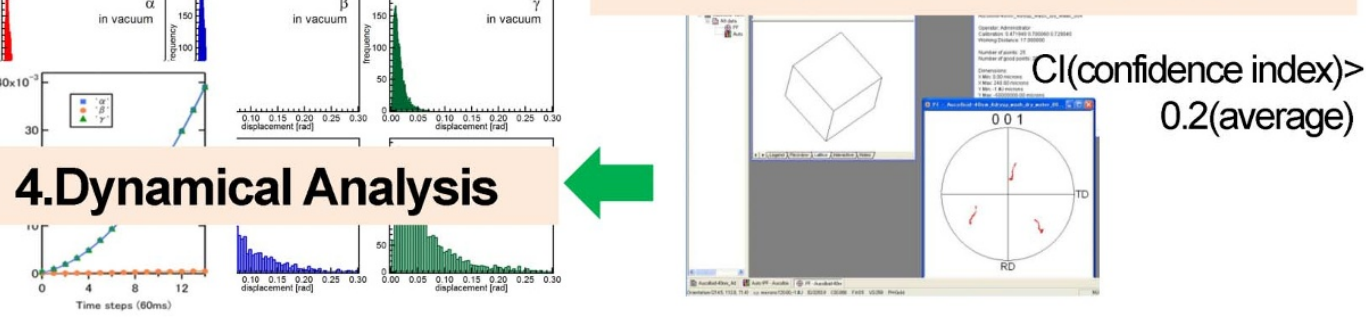

Figure $2 \mid$ DET data treatment from the labeled gold nanoparticle. DET data procedure is a simple process. (1) First, we check the positions of the adsorbed gold nanoparticle using SEM. Individual single gold nanoparticle is selected in the SEM image. (2) Next, we collect time-resolved EBSD measurements from the individual single gold nanoparticle. (3) The direction of each EBSD measurement is determined in three dimensions for each time point. Only when CI (Confidence index) value is 0.2 or more, we have counted as DET data. (4) The orientation displacements at each time point can be obtained by comparing differences in the EBSD from the crystallographic orientation pattern in front of one. In each data, it deducted from the data in front of one to make displacement. These DET displacement data is expressed as tracking data (for example Fig. 3), or a statistical treatment is carried out as shown in Fig. 4, 5, and 6).
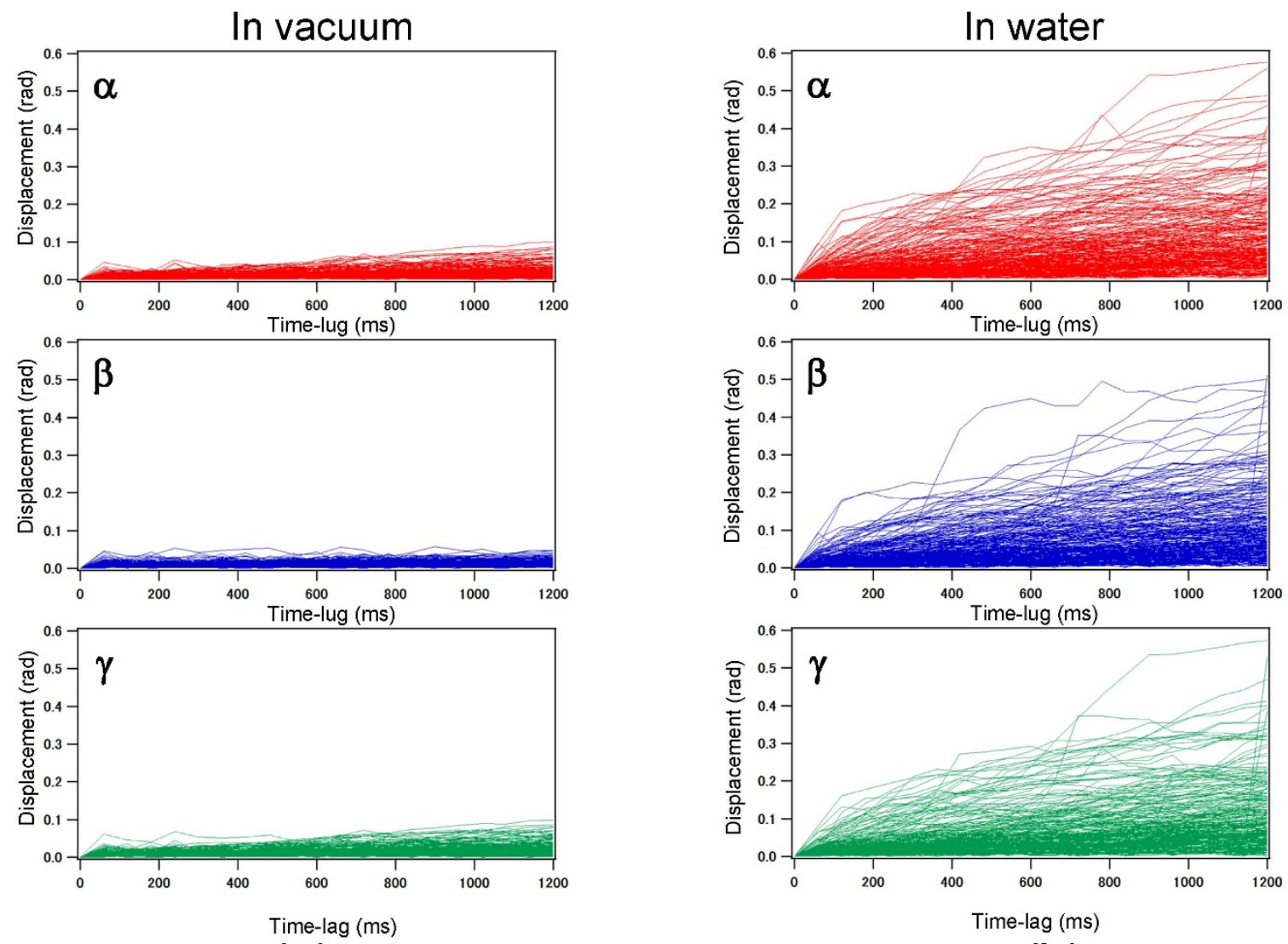

(a)

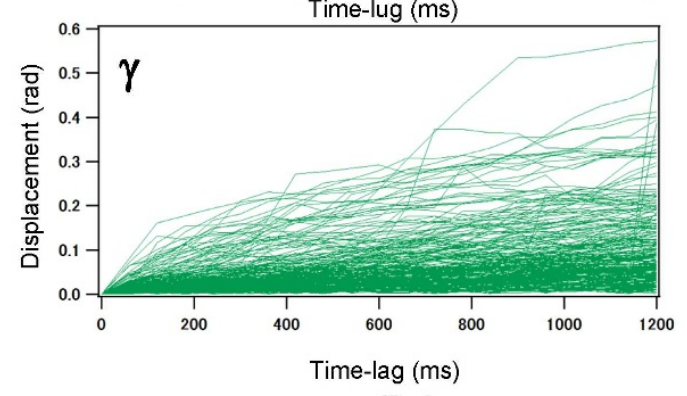

(b)

Figure $3 \mid$ (a) 3D time-resolved tracking of the individual labeled gold nanoparticles on the coating polymer film under vacuum conditions with $\alpha$, $\beta$ and $\gamma$ axes. (b) 3D time-resolved motions of the labeled gold nanoparticle on the coating polymer film in aqueous solutions with $\alpha$, $\beta$, and $\gamma$ axes. 
(a)

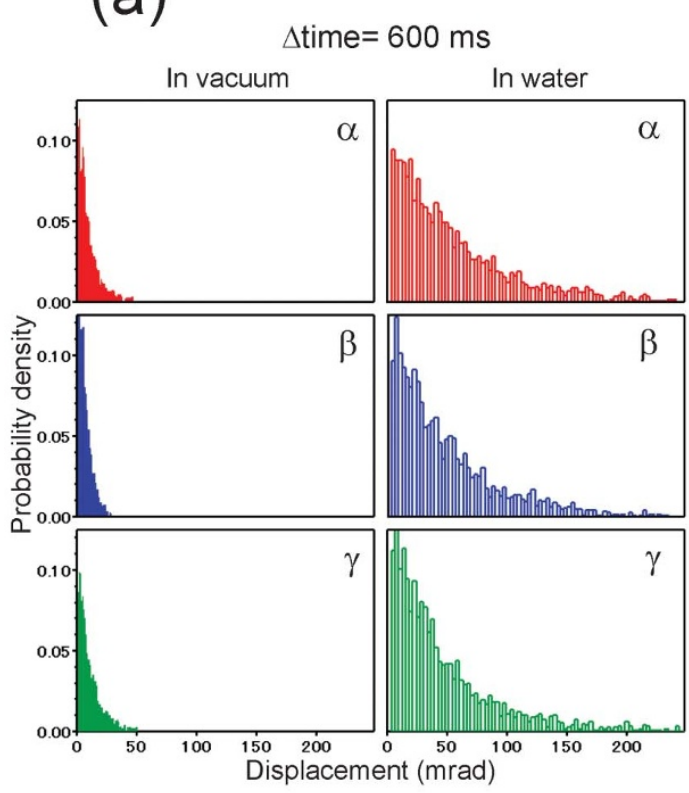

(b)

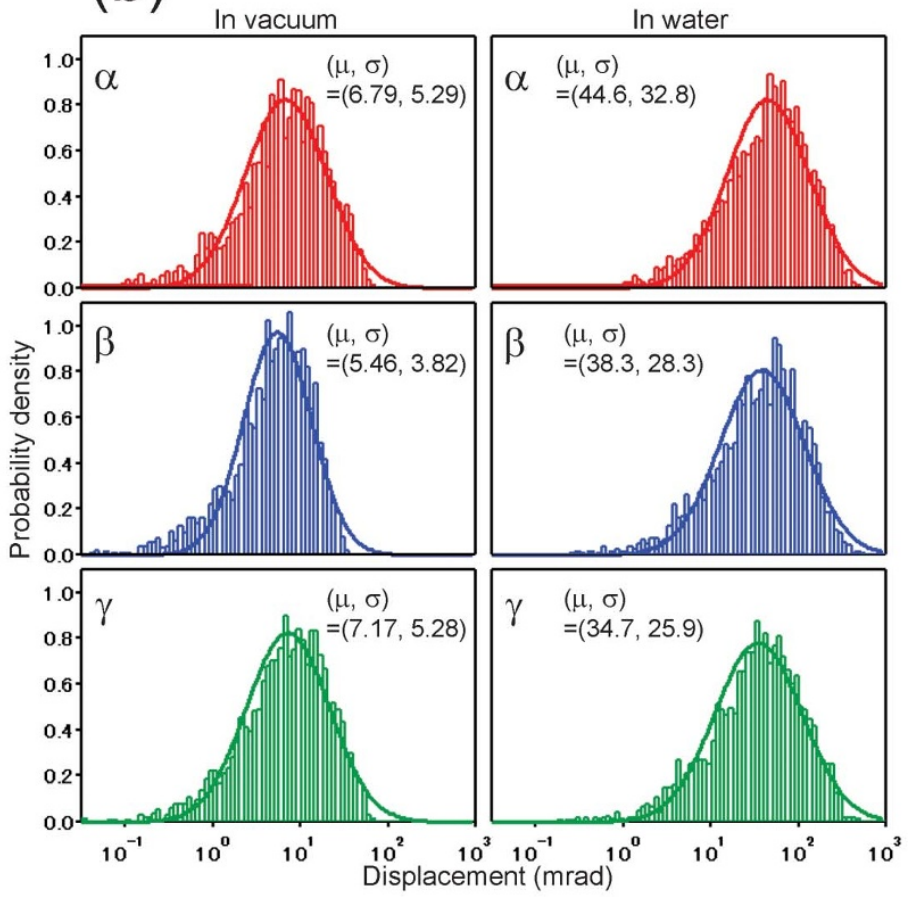

Figure $4 \mid$ (a) The 3D distributions of the observed displacements using $600 \mathrm{~ms}$ (= $\Delta 10$ frame) as the time interval. (b) A symmetrical normal Gaussian distribution using log axes can be used to characterize these log-normal distributions. These distributions are usually characterized in terms of the log-transformed displacements, using the average value, $\mu$ of its distribution and the standard deviation, $\sigma$. We confirmed that the log-normal distribution held true for many different time intervals.

(a) In vacuum $\Delta$ time $=600 \mathrm{~ms}$
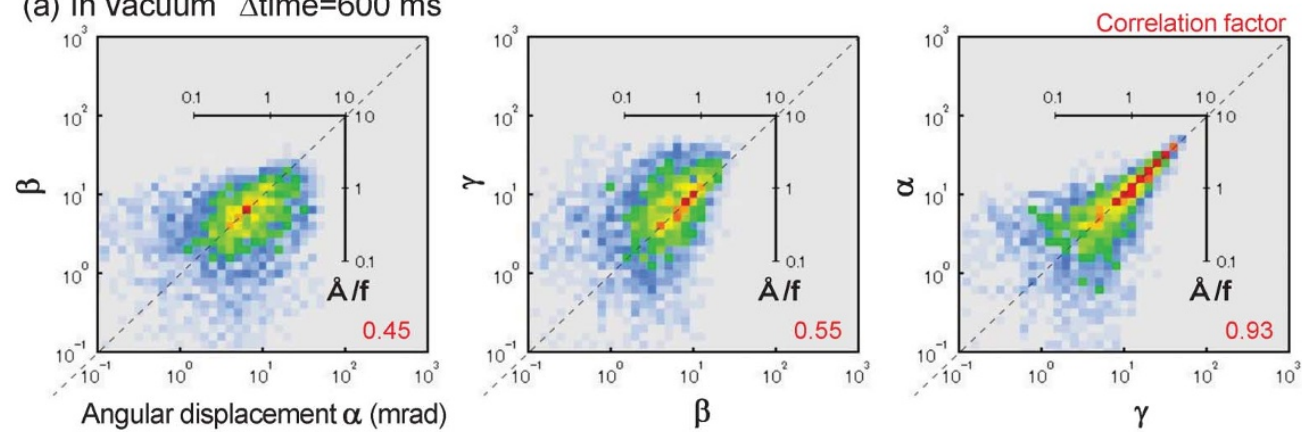

(b) In water $\Delta$ time $=600 \mathrm{~ms}$
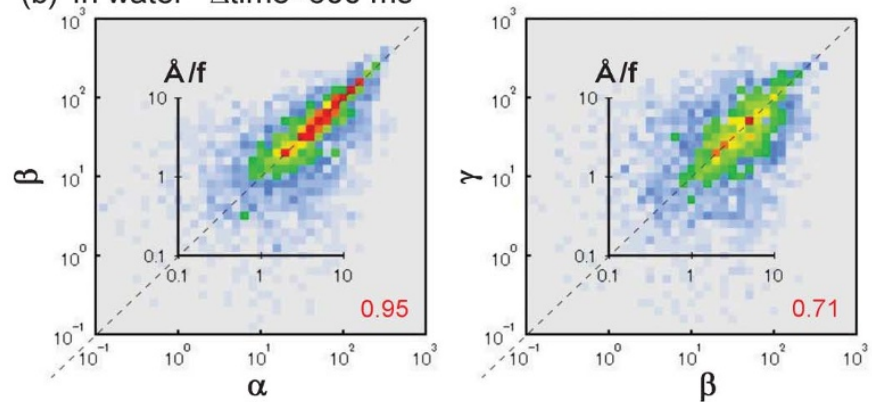

Figure 5 (a) and (b) 2D correlation histograms and the value of each correlation factors (red). From the three correlation coefficients for conditions under vacuum, we can see that the $\beta$ axis has a unique independent motility characteristic. In aqueous solutions, we can see that the $\gamma$ axis has a unique independent motility characteristic. Each inner axes means that our observed rotational movements from EBSD patterns were changed into the values of translation motions in the coating polymer surface. The unit currently used is the length (Angstrom) to one frame (Angstrom/f). It is assumed that polymer exercises in rigid body at this time. (c) $3 \mathrm{D}$ histogram of the observed displacement using $600 \mathrm{~ms}(=\Delta 10$ frame) as the time interval. The observations of 3D orientation motions in a labeled nanoparticle are realizable with super-high sensitivity. 
(a)

(b)
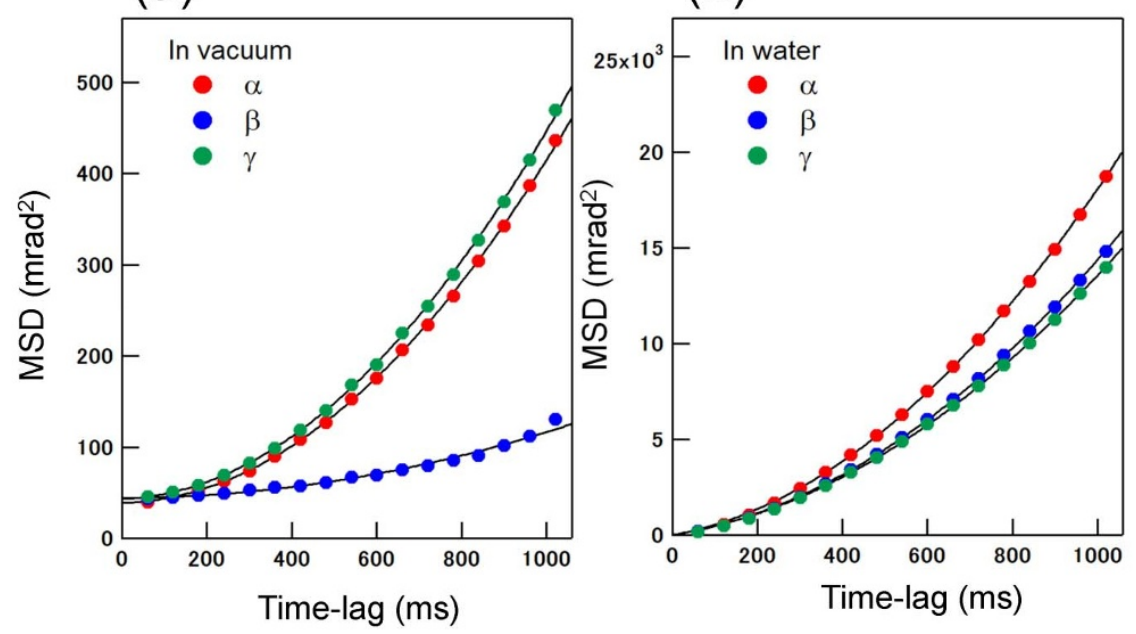

Figure 6 Plots of the mean-square displacement (MSD) against the time interval. The MSD curves of the observed gold nanoparticle as a function of time interval $\Delta \mathrm{t}$ under two conditions (under vacuum(a) and in solution(b)).

Because we observed rotation of the $\beta$-axis around the LD axis under vacuum, the dynamical data from the observed EBSD patterns were counted as small $\beta$ motions. The movement correlations between the other two axes ( $\alpha$ and $\gamma$ ) produce two-dimensional histograms (Fig. 5). In Figure 5(a), we can see from the three correlation coefficients that under vacuum conditions the $\beta$-axis is independent and has lower mobility.

The movement characteristics in aqueous solutions can be inferred from the two-dimensional histograms shown in Figure 5(b). Although it is not clear for aqueous conditions, the three correlation coefficients imply that the independent lower mobility is present in the rotational movement around the $\gamma$-axis. As shown in Figure 5(b), it is clear that both $\gamma$-direction coefficients are relatively small.

\section{Discussion}

To characterize the Brownian motion, we used plots of the meansquare displacement (MSD) against the time interval. Figures 6(a) and 6(b) show the MSD curves for the observed gold nanoparticles as a function of the time interval $\Delta t$ under vacuum and in solution. For simple Brownian diffusion, the MSD plots are linear with a slope of $4 D$, where $D$ is the diffusion coefficient. These parabolic MSD plots, however, are indicative of directed diffusion ${ }^{2}$. These data demonstrate that gold nanoparticles move at a constant drift velocity $v$ with a diffusion coefficient $D^{12}$. However, we are only concerned with the size of $D$. Under vacuum the smallest rotational motions occur around the $\beta$-axis (Fig. 6(a)). Under aqueous conditions the smallest rotational motions occur around the $\gamma$ axis (Fig. 6(b)).

We also considered why the dynamical movements differed between the vacuum and aqueous conditions. Figure 1(b) shows the arrangement of the electron beam position and an adsorbed gold nanoparticle. Under vacuum, slight anisotropic movements of the gold nanoparticles were induced by the difference between the position of the primary electron beam and the center of the gold nanoparticle. These anisotropic movements are obscured by motions that occur due to collisions between water molecules and gold nanoparticles. These collisions also explain why the adsorbed gold nanoparticle motions in aqueous solutions are 5-7 times larger than those under vacuum. In addition, it is clear that the stiffness of the adsorbed polymer layer is completely different depending on whether it is under vacuum or in an aqueous solution. As a result, 3D isotropic movements of the adsorbed nanoparticles were observed in aqueous solutions.
The rotational movement observed by DET can be converted to translational motion by making certain assumptions, as shown in Figure 7. Figure 1(b) shows the cross-sectional view of the DET sample. The rotational gold nanoparticle motions are directly reflected in the EBSD data. If the adsorbed polymer layer on the thin carbon film (i.e., the layer from the silane coupling agent) is rigid, it can transfer the local translational movements from the upper surface of the polymer film, as shown by the yellow arrow in Figure 1(b). The transferred motion widths are shown in Figures 5(a) and 5(b). For example, the range of motion is between 50 picometers per frame and 5 nanometers per frame. Because the detection limit of DET is approximately $1.0 \mathrm{mrad}$, we estimate that the smallest detectable movements of the polymer and gold nanoparticles were between 10 and 50 picometers.

DET could detect the anisotropic movement around the LD axis under vacuum conditions. The difference in the movement direction was confirmed from the anisotropic movement distribution in the 0.1 - to $5.0-\AA$ range, as shown in Figure 5(a). DET allowed us to measure for the first time the delicate movement of an adsorbed

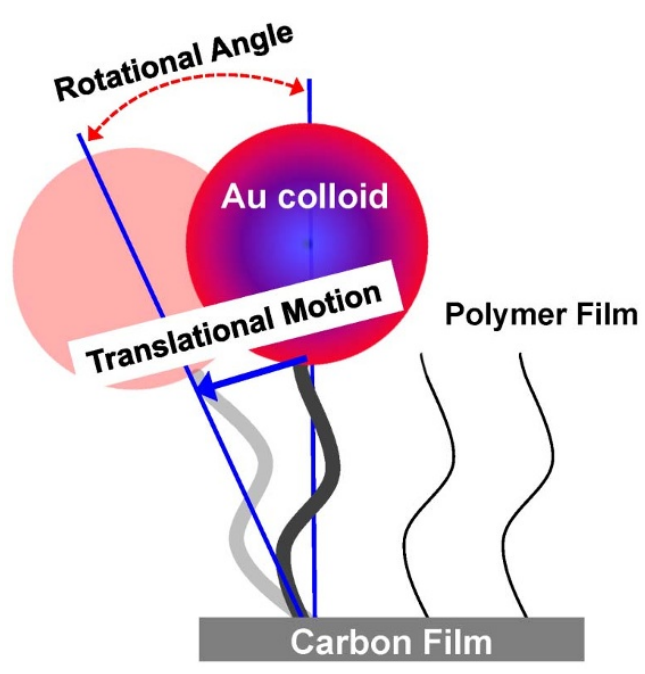

Figure $7 \mid$ DET can detect changes of a rotational angle from the observed EBSD. It is an enlargement of our DET sample. The detected rotational movement can be changed and considered to translation motion. 


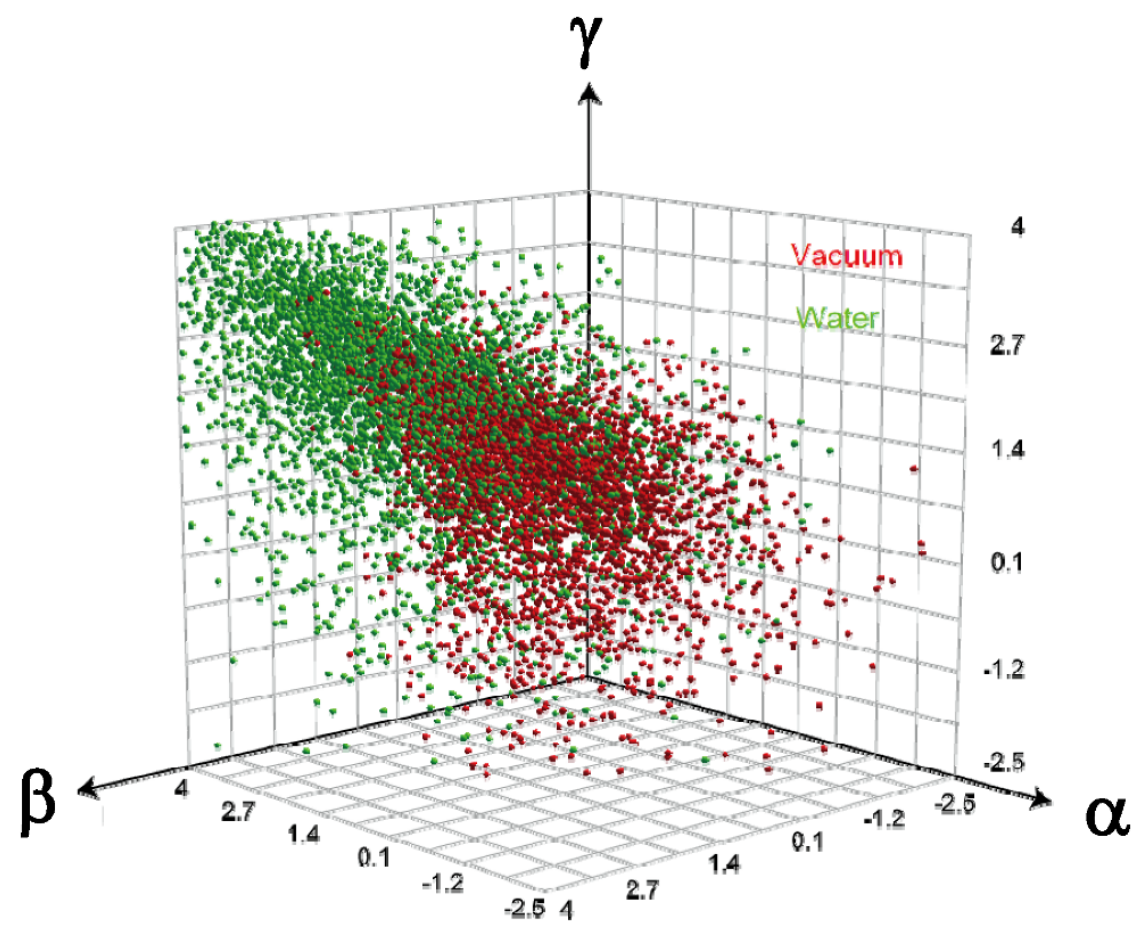

Figure 8 3D orientation motions of a labeled nanoparticle can be determined with super-high sensitivity using DET method. Figure 4 is equal to the projections to the plane of this $3 \mathrm{D}$ histogram.

nanoparticle excited by an electron probe. This movement was not from sample damage by the electron beam. The total thickness of the silane coupling agent between the adsorbed gold nanoparticle and the surface of the carbon film was approximately $100 \AA$, therefore, the slight displacements in the 0.1 - to 5.0 - $\AA$ range cannot be attributed to damage.

We were able to collect time-resolved, 3D, super-accurate (picometer scale) motion tracking data on single nanoparticles under vacuum and in solution using wet SEM and EBSD patterns. We also measured slightly excited anisotropic motions from the interactions between individual gold nanoparticles (diameter $=40 \mathrm{~nm}$ ) and the high-energy electron probe $(30 \mathrm{keV})$. This unusual motion was so small that it was obscured by Brownian motion. Because the electron beam used for DET has a short wavelength, the 3D orientation of the labeled nanoparticles can be determined with super-high sensitivity, as shown in Figure 8. When a gold nanoparticle is linked to a single protein molecule (as in DXT), a time-resolved, highly accurate, single-molecule detection system using an electron beam can be produced using normal laboratory-sized equipment (SEM equipment), without the need for a very strong X-ray source such as the SPring-8, ESRF, or APS beamlines.

\section{Methods}

Sample preparations for wet -SEM (DET). An environmental cell (EC) for SEM was designed to observe samples in aqueous environments. The EC was assembled from a special three-slit grid ( $3 \mathrm{~mm}^{2}, 0.2 \mathrm{~mm}$ thick) and an O-ring (inner diameter $2.0 \mathrm{~mm}$, thickness $0.45 \mathrm{~mm}$ ) at the top of the EC holder. The grid was designed to be used for DET at a 70 degree angle, as shown in Figure $1(\mathrm{~b})$. The slits $(0.1 \times 0.3 \mathrm{~mm})$ of the grid were covered with a thin carbon sealing film (15-20 nm thick) to prevent liquid or gas leakage from the EC. The surface of the carbon sealing film on the grid was coated with mercaptosilane reagent (KBM-803, Shinetsu silicone, Japan) using a vapor deposition method (Fig. 1(b)). A solution containing $40 \mathrm{~nm}$ colloidal gold (Polyscience, USA) was incubated on the carbon sealing film for 1 hour before washing with distilled water. For aqueous observations, the grid was made hydrophilic through a glow discharge treatment (HDT-400, JEOL, Japan). A 200-nl volume of degassed distilled water was then placed on the carbon sealing film supporting the colloidal gold. Excess water was removed by wicking with filter paper. Finally, approximately 50 to 100 nanoliters (nl) of degassed water was enclosed in the EC.
Measurement conditions for DET. For SEM studies, we used a JSM-7001F system (JEOL, Japan) equipped with a Schottky-type field emission electron gun. To detect the EBSP signal, we used a $30 \mathrm{keV}$ accelerating voltage, an $87 \mathrm{pA}$ beam current, and $17 \mathrm{~mm}$ working distance at a 70-degree angle relative to the EC sample stage. The EBSD detector on the JSM-7001F system was custom designed by TSL Solutions (DVC1412-FW-T1-EX, TSL, Japan). The detector included an image intensifier (V8070U-74, Hamamatsu Photonics, Japan), as shown in Figure S2, providing ten times higher intensity than a normal detector. We could not obtain clear EBSD data without the image intensifier. Figure 2 shows tracking of the labeled gold nanoparticles on polymer substrates under vacuum and in solution. To measure the crystal orientation of the gold nanoparticles, EBSD patterns were obtained from $1.5 \mathrm{sec}$ of electron beam irradiation at a fixed observation point. The EBSD pattern was obtained on the phosphor screen of the detector, intensified, and recorded with a CCD camera using a $60 \mathrm{~ms}$ shutter speed. The Euler angles were obtained from the EBSD patterns using OIM-data collection software (EDAX, USA). The Euler angles indicate the rotational relationship between the sample stage coordinate system and the crystal lattice coordinate system of the colloidal gold. The $\alpha, \beta$, and $\gamma$ rotational angles of the nanoparticle principal lattice vectors were calculated between adjacent frames from the Euler angles, as shown in Figure 1(a).

1. Toprak, E., Kural, C. \& Selvin, P. R. Super-Accuracy and Super-Resolution. Meth Enzymol. 475, 1-26 (2010).

2. Sasaki, Y. C. et al. Tracking of individual nanocrystals using diffracted $\mathrm{x}$ rays. Phys. Rev. E. 62(3), 3843-3847 (2000)

3. Sasaki, Y. C. et al. Picometer-Scale Dynamical X-Ray Imaging of Single DNA Molecules. Phys. Rev. Lett. 87(24), 248102-248105 (2001).

4. Sasaki, Y. C. Dynamical Observations of Soft Nanomaterials Using X-rays or High-energy Probes. 69-107, Chapter 2, Soft Nanomaterial edited by Nalwa, H. S. American Scientific Publishers (2009).

5. Thiberge, S. et al. Scanning electron microscopy of cells and tissues under fully hydrated conditions. PNAS 101(10), 3346-3351 (2004).

6. Yuk, J. M. et al. High-Resolution EM of Colloidal Nanocrystal Growth Using Graphene Liquid Cells. Science 336, 61-64 (2012).

7. Faulk, W. P. \& Taylor, G. M. An immunocolloid method for the electron microscope. Immunochem. 8(11), 1081-1083 (1971).

8. Okumura, Y., Oka, T., Kataoka, M., Taniguchi, Y. \& Sasaki, Y. C. Picometer-Scale Dynamical Observations of Individual Membrane Proteins: the case of Bacteriorhodopsin. Phys. Rev. E. 70, 021917-1-7 (2004).

9. Sagawa, T., Azuma, T. \& Sasaki, Y. C. Quantification of protein-ligand bindings from structural fluctuations of single molecules. Biochem. Biophy. Res. Commun. 335, 770-775 (2007)

10. Shimizu, H. et al. Global Twisting Motion of Single Molecular KcsA Channel upon Gating. Cell 132, 67-78 (2008). 
11. Limpert, E., Stahel, W. A. \& Abbt, M. Log-normal Distributions across the Sciences:Keys and Clues. BioScience 51, 341-352 (2001).

12. Sasaki, Y. C., Higurashi, T., Miyazaki, T., Okumura, Y. \& Oishi, N. Observations of X-ray Radiation Pressure Force on Individual Gold Nanocrystals. Appl. Phys. Lett. 89, 053121-053123 (2006).

\section{Author contributions}

Y.C.S. wrote the main manuscript text. K.H., H.S., K.I. and Y.M. analyzed DET data and prepared figures 1-6. N.O., Y.H., S.S., A.I. did main DET experiments. All authors reviewed the manuscript.

\section{Additional information}

Supplementary information accompanies this paper at http://www.nature.com/ scientificreports

Competing financial interests: The authors declare no competing financial interests.

How to cite this article: Ogawa, N. et al. Tracking 3D Picometer-Scale Motions of Single Nanoparticles with High-Energy Electron Probes. Sci. Rep. 3, 2201; DOI:10.1038/srep02201 (2013).

cc)(1) $\odot$ This work is licensed under a Creative Commons AttributionBy nc No NonCommercial-NoDerivs 3.0 Unported license. To view a copy of this license, visit http://creativecommons.org/licenses/by-nc-nd/3.0 